\title{
THEORETICAL ANALYSIS OF MAGNETORHEOLOGICAL DAMPER CHARACTERISTICS IN SQUEEZE MODE
}

\author{
Bogdan SAPIŃSKI* \\ *Department of Process Control, AGH-University of Science and Technology, \\ Al. Adama Mickiewicza 30, 30-059 Kraków, Poland \\ deep@agh.edu.pl
}

received 21 April 2015, revised 20 July 2015, accepted 22 July 2015

\begin{abstract}
The paper summarises the theoretical study of a magnetorheological (MR) damper operated in squeeze mode, intended to be used as an actuator in a semi-active mount system in a car motor. The structural design and operating principle of the damper are described and a simplified model of the MR fluid flow in the gap is presented. The plots of the damper force generated by the MR damper are obtained for monoharmonic piston motion with respect to the centre point of the gap height and in the conditions of the control coil being supplied with direct current.
\end{abstract}

Keywords: MR Damper, Squeeze Mode, Gap, Force, Characteristic

\section{INTRODUCTION}

Vibrations of the drive unit in motor vehicles are typically caused by inertia force fluctuations due to the movements of unbalanced elements in the crank mechanism and to periodic variations of torque delivered from by the motor to the drive mechanism, also due to some random forces. Vibration reduction strategies usually include the use of passive elements in the form of either rubber or hydraulic systems.

Recently, the research efforts have been focused on semiactive or active actuators intended for use in vehicle mounts. An MR damper operated in squeeze mode can serve as an actuating element (Farjoud et al, 2011; Kim J. H., 2012; Sapiński and Krupa, 2013; Zhang et al, 2011). The distinctive feature of this damper is the variable gap height and that is why the magnetic flux linked with the control circuit is dependent both on the current level and on the gap height (Gong et al, 2014; Gołdasz and Sapiński, 2011; Kim K.-J., 2008; Sapiński et al, 2015 ). Incorporating such a damper in the mount system allows the adverse effects of motor vibrations to be effectively reduced (Snamina and Sapiński, 2014).

The paper summarises the theoretical studies of an MR damper operated in the squeeze mode, basing on a simplified model of the MR fluid flow in the gap. The damper characteristics are obtained assuming the monoharmonic piston motion with respect to the current gap height centre and for the control coil being supplied with direct current. Respective plots show the time histories of damper force and its dependence on the piston displacement.

\section{DAMPER ASSEMBLY}

A schematic diagram of the damper is revealed Fig. 1. The numeric symbols in the figure indicate all key components of the damper (1-8) and the key highlights materials used for manufacturing the device. The hardware features two concentric cylinders $(1,2)$. The inner non-magnetic cylinder (2) houses the piston (3), the core assembly (4), and the floating piston (5). The core assembly incorporates the coil (6). The outer cylinder (1) material is ferromagnetic. The distance between the lower surface of the piston and the upper surface of the core is referred to as the control (working) gap of time-varying height. The distance between the piston and the core varies according to the prescribed displacement (force) input (along the vertical z-axis). The floating piston below the core assembly separates the MR fluid from the coil spring located in the compensating chamber below the floating piston (5). The chamber incorporates a preloaded spring system (not shown in Fig. 1) for fluid volume compensation. Alternatively, the compensating chamber could be filled in with pressurized gas. The choice of the coil spring was due to convenience. The current in the control coil induces a magnetic field of the strength $\mathrm{H}$. The magnetic flux that is generated by the current in the control coil (6) travels through the core and into the control gap, the outer cylinder, and back into the core through radially projected arms in the core base. The inner cylinder (2) of sufficient wall thickness is used to reduce the amount of magnetic flux bypassing the control gap, i.e. magnetic short circuit. All of the components ensure an efficient magnetic flux return path.

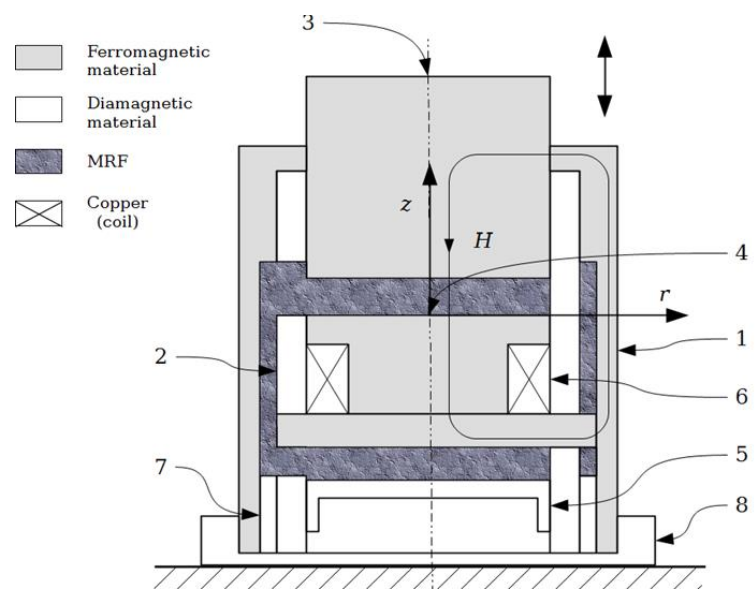

Fig. 1. Schematic diagram of the damper 
The flux that is induced in the control gap upon the application of the coil current effectively modifies the yield stress of the fluid, and its resistance to flow. As the piston moves downward, the distance between the core and the piston decreases. The excess of MR fluid is squeezed out of the control gap into the fluid volume between the inner cylinder and the outer housing of the damper, and then into the compensating chamber. The additional fluid volume that enters the compensating chamber pushes the floating piston against the coil spring. The structure incorporates a nonmagnetic ring (7), whereas the base cap (8) is used for fixing the assembly against the ground. The MR fluid type employed in the damper is BASF Basonetic 4035 (Kieburg, 2010). The outer cylinder length is $133 \mathrm{~mm}$ and the diameter is $102 \mathrm{~mm}$.

\section{MAGNETORHEOLOGICAL FLUID FLOW IN THE GAP}

MR fluid in the damper flows in the closed-loop circuit comprising the gap where the MR fluid is exposed to the magnetic field and the container in the bottom section of the damper. The gap and the container are connected by channels placed inside an inner cylinder. Downward movement of the piston at the speed $v p=\dot{h}$ induces the fluid flow away from the gap whilst during its upward motion the fluid flows towards the gap. During the piston motion the surface area (the cylinder's side wall) through which the fluid flows in and out will be changed.

Velocity of fluid elements is related to the piston velocity and its position. At small gap heights, the velocity of fluid flow tends to increase significantly. Fluid velocity distributions $v(r, z)$ in the gap are shown schematically in Fig. 2 (for the downward motion of the piston). MR fluid flow is assumed to be governed by the Bingham model whereby yield stress increases linearly with the strain rate after exceeding the yield strength, related to the magnetic field strength $H$. This model is typically recalled to describe the behaviour of MR fluids under the action of magnetic field.

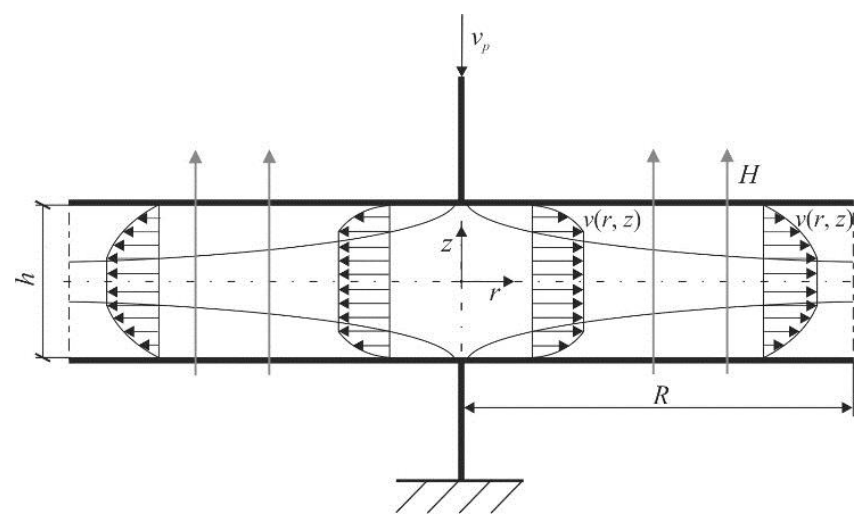

Fig. 2. Schematic diagram of MR fluid flow in the gap

Within the gap region (beneath the piston) there are three characteristic flow regimes. The first emerges near the piston surface, the second-near the cylinder bottom surface. In those regions fluid flows with the tangent stress exceeding the yield strength. As shown in velocity profiles (see Fig. 2), individual fluid layers move at speed being the function of the coordinate $z$. The third flow regime encompasses the gap's middle section, where the fluid layers do not move with respect to one another, their speed is not dependent on the coordinate $z$ and the tangent stress is less than the yield strength. On interface surfaces between those flow regimes the tangent stress equals the yield strength. In the light of the relationship between the yield strength and magnetic field strength, the locations of those interface surface as well as their actual shape will vary depending on the magnetic field strength.

\section{DAMPER CHARACTERISTICS}

Taking into account the structure of the device (see Fig. 1), the force $F$ generated by the damper has the following components:

$F=F_{\mu}+F_{\rho}+F_{\tau_{0}}+F_{S}$

where: $F_{\mu}$ - force associated with fluid viscosity, $F_{\rho} \llbracket$ - inertia force of the fluid motion, $\left(F_{\rho}=F_{\rho_{1}}+F_{\rho_{2}}\right), F_{\rho_{1}}$ - force associated with piston acceleration, $F_{\rho_{2}}$ - force related to the squared piston velocity, $F_{\tau_{0}}$ - force associated with the yield strength of fluid, $F_{S}$ - resultant force from the spring system for fluid volume compensation.

The components of the force $F$ can be expressed as:

$F_{\mu}=-\frac{3 \pi \mu R^{4}}{2 h^{3}} \dot{h}$

$F_{\rho}=F_{\rho_{1}}+F_{\rho_{2}}=-\frac{\pi \rho R^{4}}{2 h} \ddot{h}+\frac{\pi \rho R^{4}}{4}\left(\frac{\dot{h}}{h}\right)^{2}$

$F_{\tau_{0}}=-\frac{\pi R^{3} \tau_{0}}{4 h} \operatorname{sign}(\dot{h})$

$F_{S}=-k\left(h-h_{0}\right)$

where: $\mu$ - fluid viscosity, $\rho$ - fluid density, $\tau_{0}$ - yield strength, $R$ - piston diameter, $k$ - stiffness coefficient of the spring system, $h,(\dot{h}, \ddot{h})$ displacement (velocity, acceleration) of the piston, $h_{0}$ - neutral position of the spring system.

Damper characteristics are obtained basing on the geometrical data and materials specifications summarised in Sapiński (2015) and with the following assumptions:

- the gap height $h$ can vary in the range from 6.26 to $0.5 \mathrm{~mm}$,

- the piston moves with respect to the central point of the current gap height,

- piston excitation is sinusoidal with amplitude $A$ and frequency $f(z=z 0+A \sin 2 \pi f t) ;\left(z_{0}=h_{0}\right)$,

- control coil is supplied with direct current $I$.

The contribution of individual force components $F_{\mu}, F_{\rho}, F_{\tau_{0}}, F_{s}$ is examined recalling Eq (2-5) and using the MATLAB calculation data underlying the damper characteristics shown in Figs. 3-9. A thorough analysis of those characteristics allows the contributions of individual force components to be assessed in quantitative terms.

Figs. 3a, 3b, and Fig. 4 plot the relationship between the damper force and piston displacement and time histories of (in relative time $t \times f$ ) of the damper force for fluctuating piston displacement frequency and for the control coil being supplied with the current $I: 0.5 \mathrm{~A}, 2 \mathrm{~A}$. The plots clearly indicate that the current level in the control coil is the major determinant of the damper force whereas for the given current level, the frequency of piston motion (piston velocity) plays a minor role, which is best seen at small gap heights.

To examine the contributions of particular force components 
$F_{\mu}, F_{\rho}, F_{\tau_{0}}, F_{s}$ to the damper force $F$, their time histories were obtained for various piston displacements and for different current levels in the control coil. Selected plots are shown in Fig. 5, for the current $I=0.5 \mathrm{~A}$, revealing the dominant role of the force component $F_{\tau_{0}}$ associated with the yield strength of the fluid $\tau_{0}$. This term is associated chiefly with the current level in the control coil (magnetic field strength in the damper) and, in a lesser degree, with the piston velocity. These findings are confirmed by plots in Fig. 6 which compares the generated force $F$ for the current levels $I 0.5 \mathrm{~A}$ and $2 \mathrm{~A}$. It appears that the contribution of force components $F_{\mu}, F_{\rho}, F_{s}$ to the force $F$ is rather minor. Time patterns of forces $F_{\mu}, F_{\rho}, F_{s}$ obtained for other current levels and for frequency f resemble the plots in Fig. 5 . It is worthwhile to mention the term $F_{\rho}$. Fig. 7 and 8 plot the forces $F_{\rho_{1}}$ and $F_{\rho_{2}}$ in the function of piston displacement for various frequency and for the current $I=0.5 \mathrm{~A}$. It appears that those forces have unlike signs and are clearly dependent on frequency $f$ (piston velocity). The contribution of these force components to the damper force $F$ is the largest for small gap heights.

a)

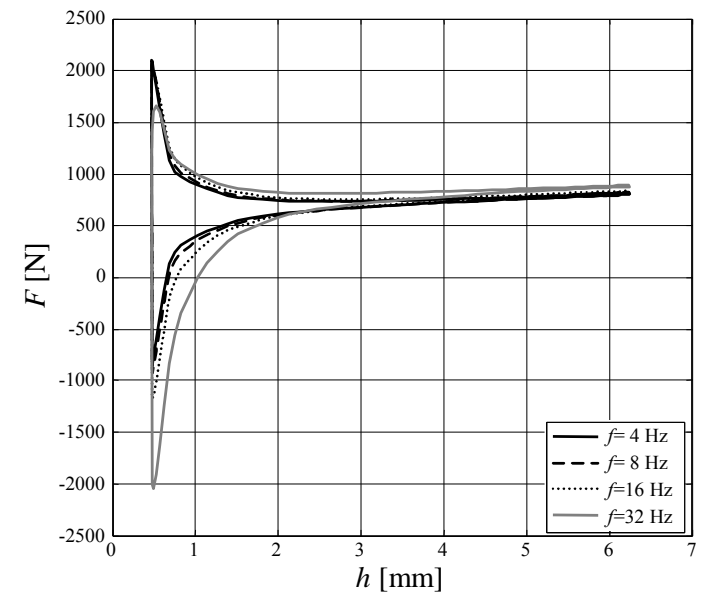

b)

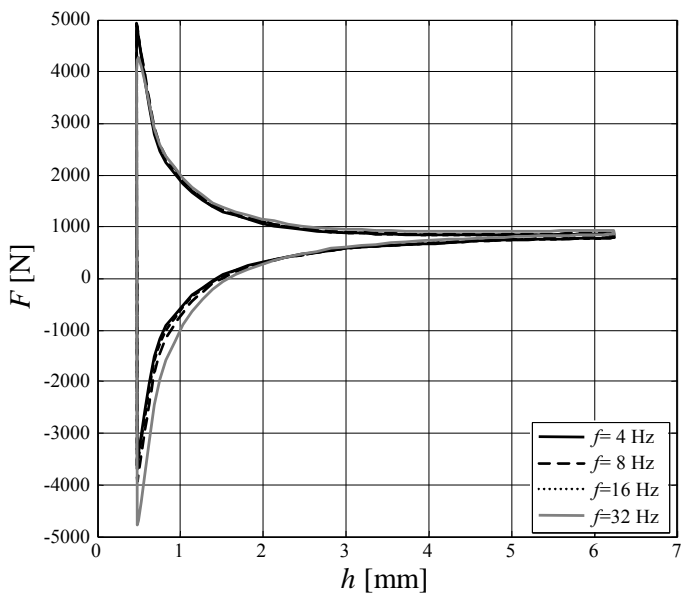

Fig. 3. Damper force vs gap height $h$ for variable frequency $f$; a) $I=0.5 \mathrm{~A}$, b) $I=2 \mathrm{~A}$

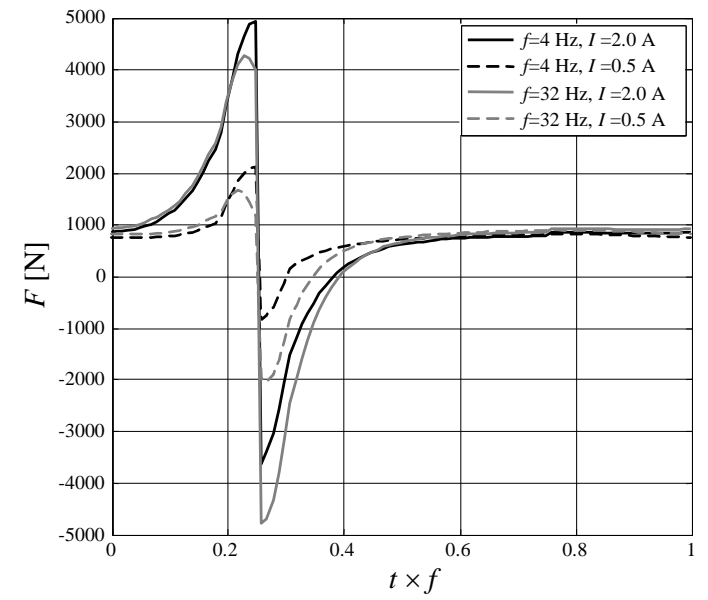

Fig. 4. Time histories of force $F$ for variable frequency $f$ and for the current $I: A=1 \mathrm{~mm}$

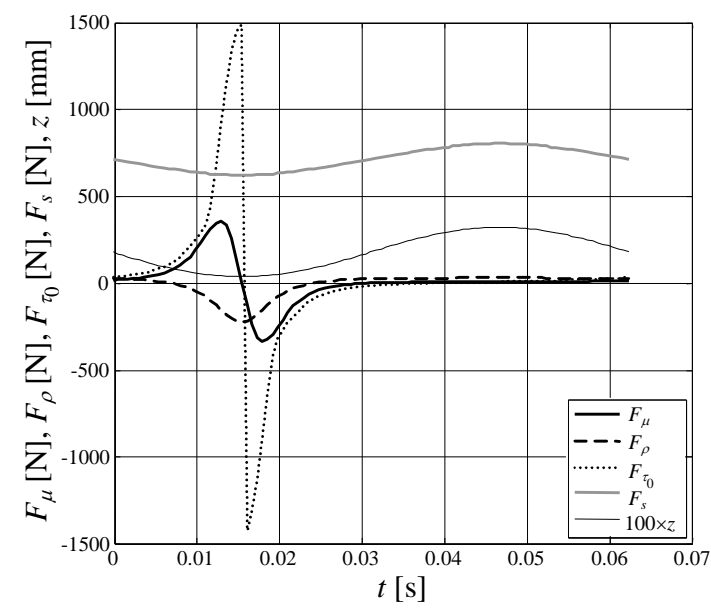

Fig. 5. Time histories of forces $F_{\mu}, F_{\rho}, F_{\tau_{0}}, F_{s}$; $f=16 \mathrm{~Hz}, A=1 \mathrm{~mm}, I=0.5 \mathrm{~A}$

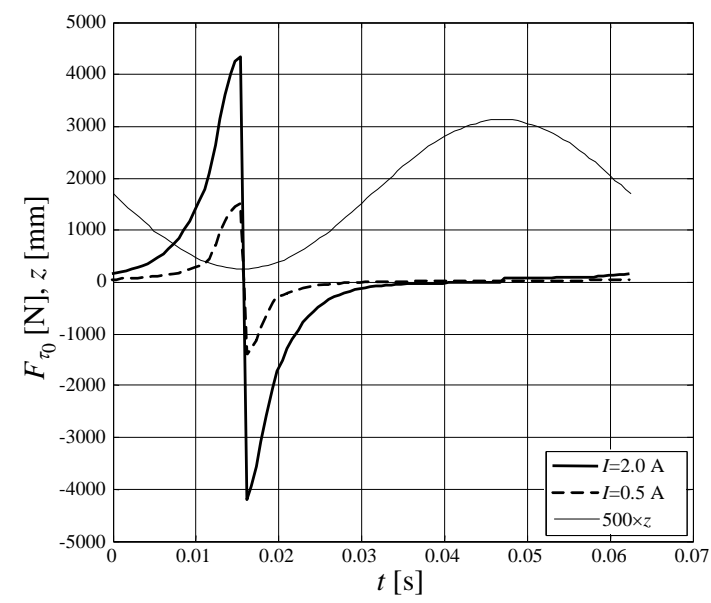

Fig. 6. Time histories of $F_{\tau_{0}}$ for various current levels $I$; $f=16 \mathrm{~Hz}, A=1 \mathrm{~mm}$ 


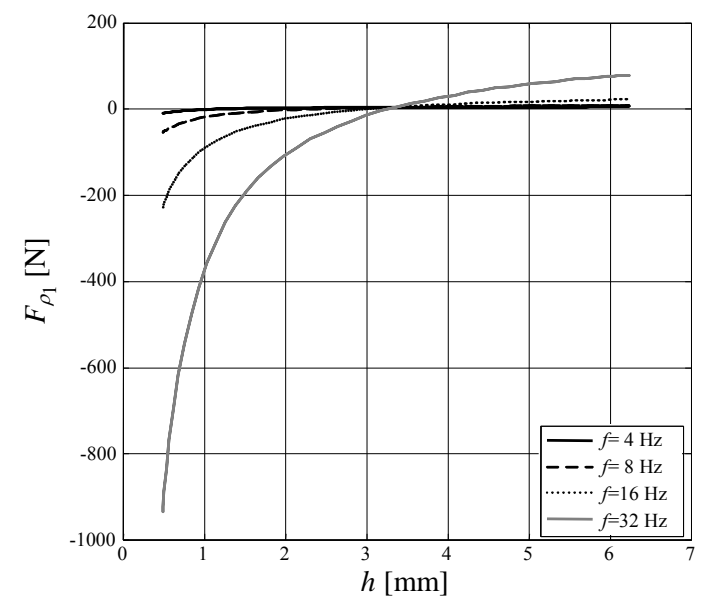

Fig. 7. Force $F_{\rho_{1}}$ vs gap height for variable frequency $f ; I=0.5 \mathrm{~A}$

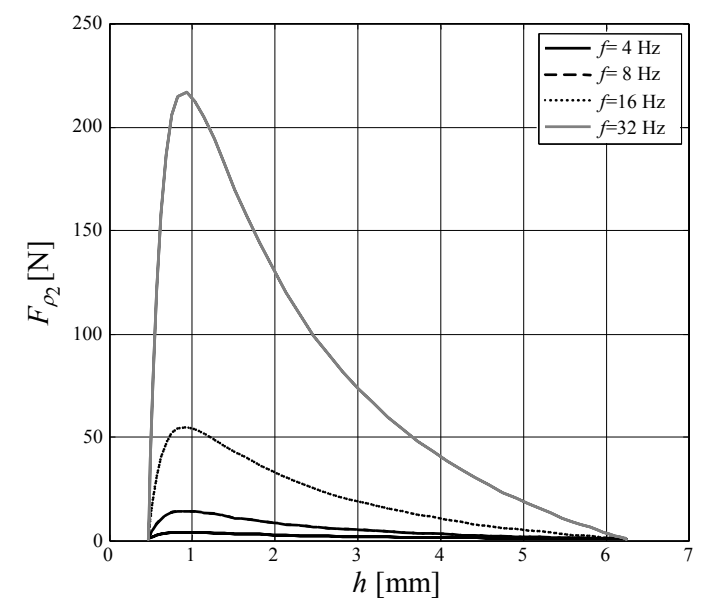

Fig. 8. Force $F_{\rho_{2}}$ vs gap height for variable frequency $f ; I=0.5 \mathrm{~A}$

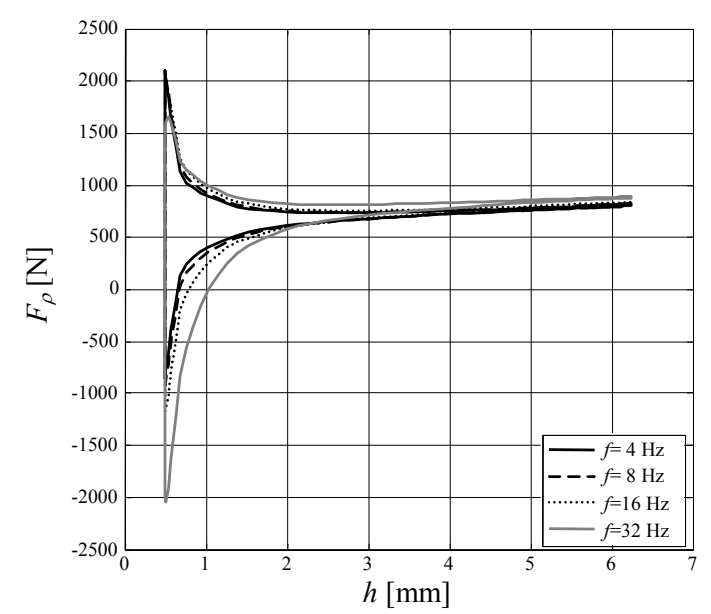

Fig. 9. Force $F_{\rho}$ vs gap height for variable frequency $f ; I=0.5 \mathrm{~A}$

\section{CONCLUSIONS}

The study is focused on theoretical characteristics of the MR damper operated in the squeeze mode. The analysis of those characteristics leads us to the following conclusions:

- of major importance is the contribution of the force component $F_{\tau_{0}}$ (associated with the fluid's yield strength and dependent on the current level in the control coil);

- for the given current level, the influence of the piston velocity on the force component $F_{\tau_{0}}$ is rather minor;

- for the given piston velocity and current level, the contribution of force components $F_{\mu}, F_{\rho}$ is minor, too;

- the influence of force components $F_{\rho_{1}}$ and $F_{\rho_{2}}$ is best revealed for small gap heights and high piston frequencies (see Figs. 7, 8).

Predicted damper characteristics will be verified by the damper prototype model testing on the tester machine.

\section{REFERENCES}

1. Farjoud A., Craft M., Burke W., Ahmadian M. (2011), Experimental investigation of MR squeeze mounts. Journal of Intelligent Material Systems and Structures, 22, 1645-1652.

2. Gołdasz J., Sapiński B. (2011), Model of a squeeze mode magnetorheological mount. Solid State Phenomena,177:116-124.

3. Gong X., Ruan X., Shouhu X., Yan, Q., Deng H. (2014), Magnetorheological Damper Working in Squeeze Mode, Hindawi Publishing Corporation, Applications of Controllable Smart Fluids to Mechanical Systems, Advances in Mechanical Engineering, Article ID 410158, $1-10$.

4. Kieburg Ch. (2010), MR Fluid Basonetic 4035, BASF Technical Information.

5. Kim J. H. (2012), Damping control device with magnetorheological fluid and engine mount having the same, United States Patent Application Publication US 2012/0132492A1.

6. Kim K.-J., Lee Ch.-W., Koo J.-H. (2008), Design and modeling of semi-active squeeze film dampers using magnetorheological fluids, Smart Materials and Structures, Vol. 17, doi: 10.1088/09641726/17/3/035006, 1-12.

7. Sapiński B. (2015), Simulation of electromagnetic phenomena in a prototype MR damper in squeeze mode, Modelling in Engineering, (to be published).

8. Sapiński B., Krupa S. (2013), Vibration isolator with MR fluid in squeeze mode, Notification of inventive design No. P.406179, 37.

9. Sapiński B., Krupa S., Matras A. (2015), Simulation of a control circuit for an MR damper in squeeze mode, Electrotechnical Review, Vol. 2015, No. 8, 135-138.

10. Snamina J., Sapiński B. (2014), Analysis of an automotive vehicle engine mount based on squeeze-mode MR damper, Technical Transactions - Mechanics, Cracow University of Technology Press, 2-M (13), 2014, 53- 63.

11. Zhang X. J., Farjud A., Ahmadian M., Guo K. H., Craft M. (2011), Dynamic Testing and Modelling of an MR Squezee Mount, Journal of Intelligent Material Systems and Structures, Vol. 22, 1717- 1728.

This research is supported by the National Centre for Research and Development under the grant No. PBS 1/A6/3/2012. 